\title{
TEORES E APORTE DE NUTRIENTES NA SERAPILHEIRA DE Pinus taeda L., E SUA RELAÇÃO COM A TEMPERATURA DO AR E PLUVIOSIDADE ${ }^{1}$
}

\author{
Márcio Viera² e Mauro Valdir Schumacher ${ }^{2}$
}

\begin{abstract}
RESUMO - O objetivo deste estudo foi avaliar os teores e o aporte de nutrientes ao piso florestal num povoamento de Pinus taeda L. e sua relação com a temperatura do ar e pluviosidade, implantado em área anteriormente ocupada por campo nativo, na cidade de Cambará do Sul, RS, através da queda de serapilheira, avaliado durante um período de três anos, contados a partir do quinto ao sétimo ano de idade do povoamento. A serapilheira foi coletada mensalmente através de coletores de $1,0 \mathrm{~m}^{2}(1,0 \times 1,0 \mathrm{~m})$, durante o período compreendido de abril/2004 a março/2007. Após cada coleta, as amostras de serapilheira foram analisadas quimicamente, quanto aos teores de macro e micronutrientes. Os teores de $\mathrm{K}, \mathrm{Mg}$ e Cu correlacionaram-se positivamente $(\mathrm{p}<0,05)$ com a pluviosidade, e os teores de $\mathrm{S}$ também o fizeram, porém com a temperatura do ar, enquanto os teores de Fe ( $\mathrm{p}<0,01)$, Mn e Zn ( $<$ 0,05) correlacionaram negativamente com essa característica climática. Os teores de $\mathrm{N}$ e K correlacionaram-se negativamente ( $\mathrm{p}<0,01$ e $\mathrm{p}<0,05$, respectivamente) com a quantidade de serapilheira depositada mensalmente. $\mathrm{O}$ aporte médio anual de macronutrientes ao solo, através da deposição da serapilheira, em $\mathrm{kg} \mathrm{ha}^{-1}$, foi de: 18,8 de Ca; 13,0 de N; 3,0 de Mg; 1,6 de K; 1,3 de S; e 1,1 de P, sendo a transferência de micronutrientes em $\mathrm{g} \mathrm{ha}^{-1}$ de: 4.708,3 de Mn; 592,3 de Fe; 74,1 de Zn; 34,0 de B; e 7,6 de $\mathrm{Cu}$. Isso demonstra a importância da permanência da serapilheira sobre o solo para manutenção da ciclagem de nutrientes.
\end{abstract}

Palavras-chave: Aporte de nutrientes, Pinus e Campo nativo.

\section{CONTENTS AND INPUT OF NUTRIENTS IN Pinus taeda L. LITTER RELATED TO AIR TEMPERATURE AND RAINFALL}

\begin{abstract}
The objective of this study was to evaluate the contents and input of nutrients to forest floor in a Pinus taeda $L$. stand and the relation with rainfall and air temperature, in an area previously occupied with native grass, in Cambará do Sul, RS, through litterfall, evaluated during a three-year period, from the $5^{\text {th }}$ to the $7^{\text {th }}$ year of the stand. Litter was monthly collected in $1.0 \mathrm{~m}^{2}(1.0 \times 1.0 \mathrm{~m})$ collectors, from Aprill 2004 to March/2007. After each collection, litter samples were chemically analyzed for macro and micronutrients. The contents of $K, \mathrm{Mg}$ and $\mathrm{Cu}$ showed a positive correlation $(p<0.05)$ with air temperature, while $\mathrm{Fe}(p<0.01)$, $M n$ and $Z n(p<0.05)$ showed a negative correlation with this climatic variable. The contents of $N$ and $K$ showed a negative correlation ( $p<0.01$ e $p<0.05$, respectively). The contents of $K, \mathrm{Mg}$ and $\mathrm{Cu}$ showed a positive correlation ( $p<0.05)$ with rainfall and the contents of $S$ showed a positive correlation $(p<0,05)$ with air temperature, while the contents of Fe ( $p<0.01), M n$ and $\mathrm{Zn}(p<0.05)$ showed a negative correlation with this variable. The contents of $N$ and $K$ showed a negative correlation $(p<0.01$ and $p<0.05$, respectively) with litter amount monthly deposited. Macronutrient mean annual input to soil, through litter deposition, in $\mathrm{kg} \mathrm{ha}^{-1}$ was: 18.8 of $\mathrm{Ca} ; 13.0$ of $\mathrm{N} ; 3.0$ of $\mathrm{Mg} ; 1.6$ of $\mathrm{K} ; 1.3$ of $\mathrm{S}$ and 1.1 of $\mathrm{P}$ and for micronutrients

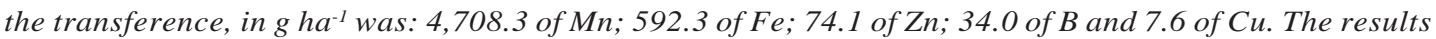
demonstrate the importantance of keeping the litter over the soil to maintain nutrient cycling.
\end{abstract}

Key-words: Nutrients input, Pinus and Native grass.

\footnotetext{
${ }^{1}$ Recebido em 11.03.2008 e aceito para publicação em 14.10.2009.

${ }^{2}$ Universidade Federal de Santa Maria, Departamento de Ciências Florestais - Santa Maria, RS - Brasil. E-mail: <vieraflorestal@yahoo.com.br>e<mvschumacher@gmail.com>.
} 


\section{INTRODUÇÃO}

O conhecimento do aporte de nutrientes através da serapilheira é extremamente importante para avaliar o impacto e implicações das ações de manejo na disponibilidade de nutrientes no solo e a produtividade futura dos povoamentos florestais (FERREIRA et al., 2004; VITAL et al., 2004). A serapilheira contém grande proporção dos nutrientes extraídos do solo pelas árvores, e, à medida que o material decíduo vai-se decompondo, os nutrientes nele contidos vão sendo liberados, dando sequência à ciclagem de nutrientes (planta-solo- planta) (KOEHLER et al., 1987; SCHUMACHER et al., 2004), que são passíveis de serem reabsorvidos pelas raízes das plantas (POGGIANI e SCHUMACHER, 2000; SCHUMACHER et al., 2003).

O processo de ciclagem de nutrientes num ecossistema florestal abrange as trocas de elementos minerais entre os seres vivos e o ambiente que os circunda, centrando-se nas relações entre a vegetação e o solo. Essa troca de nutrientes de um compartimento para outro abrange uma série de eventos, caracterizada em três formas: ciclo geoquímico (trocas de elementos minerais entre diferentes ecossistemas), ciclo biogeoquímico ou biológico (trocas químicas entre solo e planta) e ciclo bioquímico (translocação de nutrientes, antes da abscisão foliar, dentro da própria planta) (PRITCHETT, 1979; SCHUMACHER et al., 2004).

Os elementos minerais são, dessa forma, continuamente transferidos entre os compartimentos bióticos e os abióticos. Por meio do estudo dessa transferência, obtêm-se informações sobre a distribuição de nutrientes no ecossistema, podendo-se inferir sobre os fluxos entre os diferentes compartimentos (GOLLEY, 1983; JORDAN, 1985). Esse fluxo de nutrientes supre grande parte das necessidades das árvores (FERREIRA et al., 2004).

Para Hagg (1985), Britez et al. (1992), Melo e Resck (2002), Balieiro et al. (2004) e Vital et al. (2004), a ciclagem de nutrientes através da queda de serapilheira é o meio mais importante para a nutrição dos vegetais, sobretudo em solos com baixo teor de nutrientes, os quais geralmente são destinados para o cultivo de espécies florestais, e estas se desenvolvem em decorrência da queda de componentes senescentes da parte aérea, os quais, depois de serem decompostos, podem liberar quantidades significativas de nutrientes no solo. Isso é, muitas vezes, evidenciado em florestas estabelecidas em áreas contendo solos de baixa fertilidade e incapazes de suportar outras culturas.

Os principais mecanismos responsáveis pela transferência de nutrientes da biomassa de espécies arbóreas para o solo são: a deposição de serapilheira, a lavagem da vegetação pela chuva que carrega substâncias minerais e orgânicas presas às estruturas da parte aérea e a decomposição da biomassa morta, que inclui a serapilheira e raízes mortas (SANCHEZ, 1976; GONZALEZ e GALLARDO, 1986).

A concentração e o conteúdo de nutrientes na serapilheira variam em função do tipo de solo, da vegetação, da densidade populacional, da habilidade da espécie em absorver, utilizar e translocar os nutrientes antes da senescência foliar, da proporção de folhas em relação aos demais componentes, do habitat (condições edafoclimáticas) e da idade das árvores (KOEHLER, 1989; SCHUMACHER, 1992; NEVES et al., 2001). Segundo Pritchett (1979), a absorção dos nutrientes pelas árvores é influenciada pela espécie, pela cobertura florestal e pelas condições de solo e clima. Em princípio, a absorção anual de nutrientes pela maioria das espécies florestais é da mesma ordem da apresentada pelas culturas agrícolas, mas, como a maior parte dos nutrientes absorvidos é devolvida para o piso florestal, quantidades relativamente pequenas são retidas no acréscimo anual da biomassa arbórea. No entanto, segundo Dias et al. (2002), estudos sobre ciclagem de nutrientes em ecossistemas florestais são inúmeros, mas poucos levam em consideração a variação temporal da concentração de nutrientes na serapilheira e a sua relação com o clima (temperatura e precipitação). Para Brun et al. (2001), a influência do clima é determinante na quantidade serapilheira depositada em uma floresta, bem como na variação da concentração de nutrientes.

Em períodos de maior pluviosidade, as plantas poderiam apresentar elevação dos teores de nutrientes em seus tecidos, devido á maior absorção de alguns nutrientes, colocando-os plenamente disponíveis em todas as partes das plantas (DIAS et al., 2002). Já Schumacher et al. (2004) salientaram que no verão, em parte devido à maior atividade fisiológica das plantas, aumentam-se as taxas de alocação de nutrientes, o que contribui para o crescimento da planta nesse período e, consequentemente, aumenta-se a translocação de nutrientes no interior da planta. 
Em razão desse fato, o objetivo deste trabalho foi determinar os teores e o aporte de nutrientes, em Pinus taeda L., através da queda de material senescente (litter), e suas relações com a temperatura do ar e a pluviosidade, durante um período de três anos, na região de Cambará do Sul, RS.

\section{MATERIAL E MÉTODOS}

\subsection{Caracterização da área experimental}

Este estudo foi realizado em um povoamento de Pinus taeda L., em área pertencente à empresa Reflorestadores Unidos, na cidade de Cambará do Sul, RS, situada nas coordenadas geográficas de $29^{\circ} 10^{\prime}$ de latitude sul e $50^{\circ} 19^{\prime}$ de longitude oeste, numa altitude média de $893 \mathrm{~m}$. A cidade de Cambará do Sul localiza-se no Nordeste do Estado do Rio Grande do Sul, na região fisiográfica dos Campos de Cima da Serra. O tipo de clima predominante na região, segundo a classificação climática de Köppen, é o Cfb (temperado úmido), com uma precipitação média em torno de $1.700 \mathrm{~mm}$, bem distribuída durante o ano. A temperatura média anual é de aproximadamente $15^{\circ} \mathrm{C}$, sendo a média das temperaturas máximas de $22^{\circ} \mathrm{C}$ e a média das temperaturas mínimas de $8,5^{\circ} \mathrm{C}$ (MORENO, 1961). Os ventos dominantes na região são alísios, ou seja, sopram do mar para a terra, situação atribuída à proximidade da região $(60 \mathrm{~km})$ do litoral Norte do Estado do Rio Grande do Sul (MOTTA et al., 1971).

O solo local é originário de rocha basáltica, resultante do derrame basáltico do Triássico Superior, com predomínio de solos rasos com horizonte A de coloração escura, com baixa saturação de bases e teor elevado de alumínio trocável (BRASIL, 1973). O solo da área experimental pertence à Unidade de Mapeamento Bom Jesus, sendo classificado como Cambissolo Húmico alumínico típico (STRECK et al., 2002). As principais características químicas do solo sob a floresta de Pinus taeda encontram-se na Tabela 1. Essas informações foram obtidas no estudo de Schumacher et al. (2007), os quais realizaram coletas de solo nas profundidades de: 0-10, 10-20, 20-30, 30-40, 40-50 e 50-80 cm, nas quatro parcelas experimentais onde estavam localizados os coletores de serapilheira.

O experimento com Pinus taeda foi implantada em área anteriormente ocupada com campo nativo. Para a implantação do Pinus taeda, escarificou-se o solo na linha de plantio até uma profundidade média de $30 \mathrm{~cm}$. O plantio das mudas foi efetuado no período de inverno do ano de 1999, utilizando-se clones de Pinus taeda, no espaçamento de 3,0 x 2,0 m. No plantio não foi adicionada nenhuma fonte de nutrientes ao solo e, ainda, não se fizeram manutenções no plantio, como: controle de matocompetição e de pragas. Aos 4 anos de idade, realizou-se a desrama das árvores até $2,5 \mathrm{~m}$ de altura, a qual se caracterizou como a única interferência no povoamento de pinus.

\subsection{Metodologia do estudo}

Para a coleta de material senescente (serapilheira), alocaram-se, de forma aleatória (SOARES et al., 2006), quatro parcelas de 18 x 50 m, na área experimental. Em cada parcela foram distribuídos cinco coletores quadrangulares, com bordas de madeira, profundidade de $20 \mathrm{~cm}$, fundo em tela de náilon de $1 \mathrm{~mm}$ e área útil de coleta de $1,0 \mathrm{~m}^{2}(1,0 \times 1,0 \mathrm{~m})$, dispostos a $0,70 \mathrm{~m}$ de altura em relação ao piso florestal, num total de 20 coletores em todo o povoamento.

A serapilheira acumulada nos coletores foi coletada mensalmente (no dia 20 de cada mês, para estabelecer um padrão estacional), durante o quinto,

Tabela 1 -Características químicas do solo na área experimental, Cambará do Sul,- RS.

Table 1 - Soil chemical characteristics in the experimental area, Cambará do Sul, - RS.

\begin{tabular}{|c|c|c|c|c|c|c|c|c|c|c|c|c|}
\hline \multirow[t]{2}{*}{ Prof.(cm) } & M.O. & Arg & \multirow{2}{*}{$\frac{\text { CTC }}{\text { efetiva }}$} & \multirow{2}{*}{$\frac{\mathrm{pH}}{\mathrm{CaCl}_{2}}$} & $\mathrm{Al}$ & $\mathrm{Ca}$ & \multirow[t]{2}{*}{$\mathrm{Mg}$} & $\mathrm{P}$ & $\mathrm{K}$ & $\mathrm{Cu}$ & $\mathrm{Fe}$ & Mn \\
\hline & \multicolumn{2}{|c|}{$\%$} & & & \multicolumn{2}{|c|}{$\mathrm{cmol}_{\mathrm{c}} \mathrm{dm}^{-3}$} & & \multicolumn{5}{|c|}{$\mathrm{mg} \mathrm{dm}^{-3}$} \\
\hline $0-10$ & 4,4 & 44,5 & 6,7 & 3,68 & 5,5 & 0,6 & 0,5 & 2,7 & 35,3 & 2,4 & 104,8 & 15,7 \\
\hline $10-20$ & 4,3 & 43,5 & 6,4 & 3,71 & 5,6 & 0,4 & 0,3 & 2,0 & 22,2 & 2,4 & 80,0 & 3,3 \\
\hline $20-30$ & 4,0 & 51,5 & 6,2 & 3,72 & 5,6 & 0,4 & 0,2 & 1,5 & 20,7 & 2,4 & 67,7 & 2,7 \\
\hline $30-40$ & 3,5 & 52,4 & 5,8 & 3,75 & 5,4 & 0,3 & 0,2 & 1,1 & 17,7 & 2,5 & 54,3 & 1,0 \\
\hline $40-50$ & 2,8 & 55,0 & 6,0 & 3,75 & 5,5 & 0,3 & 0,1 & 1,2 & 15,3 & 2,5 & 51,7 & 2,0 \\
\hline $50-80$ & 2,3 & 58,3 & 6,1 & 3,76 & 5,7 & 0,2 & 0,1 & 1,1 & 15,3 & 2,4 & 44,9 & 3,6 \\
\hline
\end{tabular}

Fonte: SCHUMACHER et al., ( 2007).

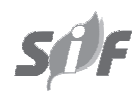

R. Árvore, Viçosa-MG, v.34, n.1, p.85-94, 2010 
sexto e sétimo anos de idade da floresta (abril de 2004 a março de 2007). O material recolhido foi acondicionado em embalagem plástica devidamente identificada e, posteriormente, enviado ao Laboratório de Ecologia Florestal do Departamento de Ciências Florestais da Universidade Federal de Santa Maria, no Rio Grande do Sul.

No laboratório, as amostras foram secas em estufa de circulação forçada de ar a $70^{\circ} \mathrm{C}$ por $72 \mathrm{~h}$, para determinação do peso seco, o qual foi estabelecido em balança digital de precisão $(0,01 \mathrm{~g})$. Para as análises químicas, as amostras provenientes dos cinco coletores de cada parcela foram misturadas, formando uma amostra composta por parcela, e, posteriormente, moídas em moinho tipo Wiley com peneira de 30 mesh. Nessas amostras foram determinados os teores de $\mathrm{N}$ pelo método Kjeldahl, $\mathrm{P}$ e B por espectrometria visível, $\mathrm{K}$ por fotometria de chama, $\mathrm{S}$ por turbidimetria e o $\mathrm{Ca}, \mathrm{Mg}, \mathrm{Cu}, \mathrm{Fe}, \mathrm{Mn}$ e Zn por espectrofotometria de absorção atômica, seguindo metodologia descrita por Tedesco et al. (1995).

O peso seco médio mensal da serapilheira e o teor de nutrientes nela contido permitiram estimar o aporte médio mensal de nutrientes ao piso florestal. Todas as análises estatísticas foram realizadas com o auxílio do software SPSS 7.5.1 for Windows (1996) no nível de $5 \%$ de probabilidade, tanto para a comparação de médias através do teste de Tukey quanto para a análise de correlação de PEARSON (r).

As informações sobre a pluviosidade $(\mathrm{mm})$ e a temperatura média do ar $\left({ }^{\circ} \mathrm{C}\right.$ ) da região (Figura 1 ), no período de estudo, foram disponibilizadas pela Estação Meteorológica do $8^{\circ}$ Distrito de Meteorologia ( $8^{\circ}$ DISME) do Instituto Nacional de Meteorologia (INMET), localizada no Município de Cambará do Sul, RS.

\section{RESULTADOS}

Entre os macronutrientes, Ca e $\mathrm{N}$ foram os que apresentaram maiores teores, em termos médios, na serapilheira (Tabela 2), seguidos, em ordem decrescente, por $\mathrm{Mg}>\mathrm{K}>\mathrm{S}>\mathrm{P}$. Coeficiente de variação superior a $20 \%$ foi constatado para K e S.

Nitrogênio e potássio apresentaram maiores teores no período primavera-verão e os menores no outono, correlacionando-se com a quantidade de serapilheira acumulada $(\mathrm{r}=-0,72 ; \mathrm{p}<0,01$ e $\mathrm{r}=-0,62$; e $\mathrm{p}<0,05$, respectivamente). Ressalta-se que o K também demonstrou correlação com a pluviosidade $(r=0,60$; e $p<0,05)$.

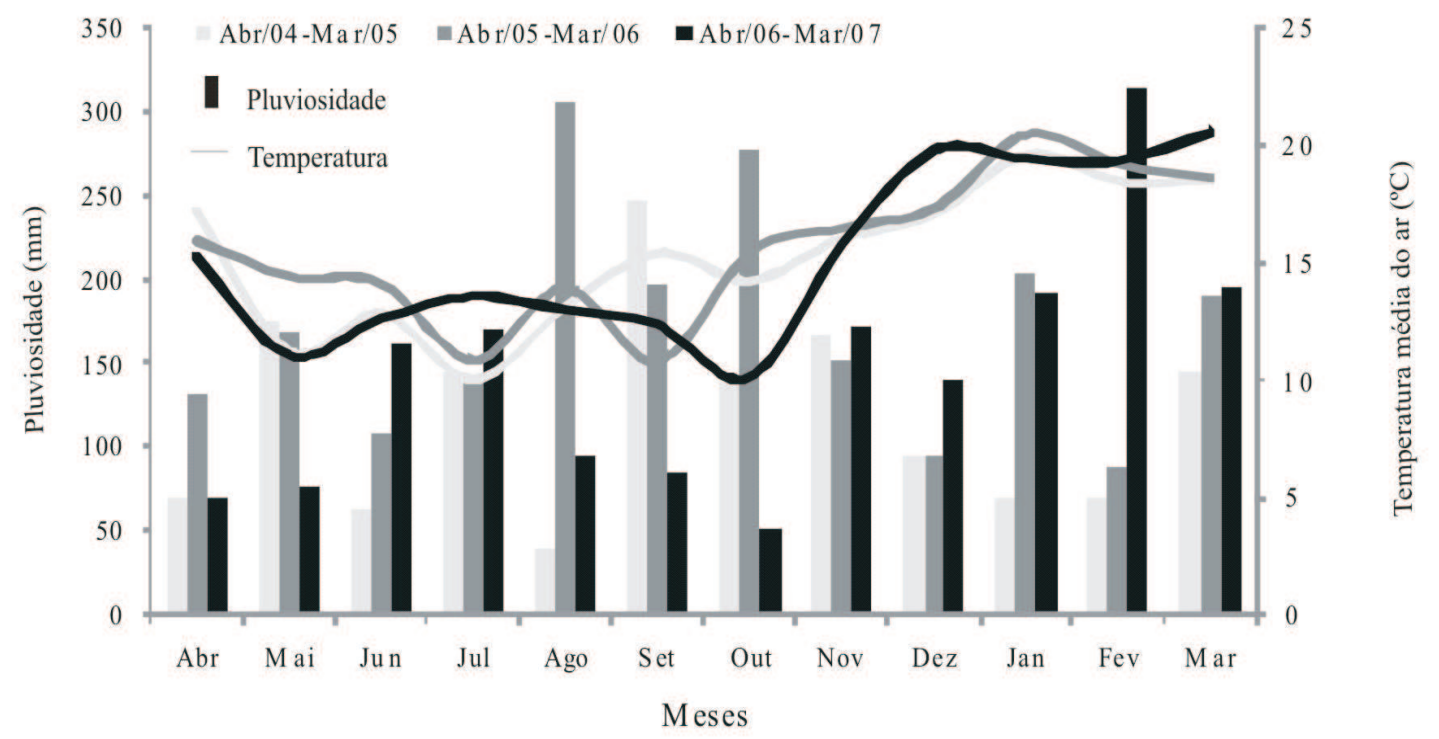

Figura 1 - Variação mensal da pluviosidade $(\mathrm{mm})$ e da temperatura do ar $\left({ }^{\circ} \mathrm{C}\right)$ durante o período de estudo, em Cambará do Sul, RS.

Figure 1-Monthly rainfall variation ( $\mathrm{mm}$ ) and air temperature $\left({ }^{\circ} \mathrm{C}\right)$ during the study period in Cambará do Sul, RS.

R. Árvore, Viçosa-MG, v.34, n.1, p.85-94, 2010 
Tabela 2 - Teor médio mensal e anual de macronutrientes na serapilheira de um povoamento de Pinus taeda. Abr./2004 a Mar./2007. Cambará do Sul, RS.

Table 2 - Monthly and annually mean macronutrient content in Pinus taeda stand litter, April 2004 to March, 2007. Cambará do Sul, RS.

\begin{tabular}{|c|c|c|c|c|c|c|}
\hline \multirow[t]{2}{*}{ Meses } & \multicolumn{6}{|c|}{ Macronutrientes $\left(\mathrm{g} \mathrm{kg}^{-1}\right)$} \\
\hline & $\mathrm{N}$ & $\mathrm{P}$ & $\mathrm{K}$ & $\mathrm{Ca}$ & $\mathrm{Mg}$ & $\mathrm{S}$ \\
\hline Abril & $\begin{array}{c}4,32 \mathrm{~d}^{\backslash 1} \\
( \pm 0,31)^{12}\end{array}$ & $\begin{array}{l}0,41 \mathrm{bc} \\
( \pm 0,07)\end{array}$ & $\begin{array}{l}0,50 \mathrm{~cd} \\
( \pm 0,14)\end{array}$ & $\begin{array}{l}6,67 \mathrm{bc} \\
( \pm 1,03)\end{array}$ & $\begin{array}{c}1,04 \text { a } \\
( \pm 0,15)\end{array}$ & $\begin{array}{l}0,40 \mathrm{~cd} \\
( \pm 0,13)\end{array}$ \\
\hline Maio & $\begin{array}{l}4,28 \mathrm{~d} \\
( \pm 0,30)\end{array}$ & $\begin{array}{l}0,41 \mathrm{bc} \\
( \pm 0,07)\end{array}$ & $\begin{array}{l}0,48 \mathrm{~d} \\
( \pm 0,12)\end{array}$ & $\begin{array}{l}6,21 \mathrm{bc} \\
( \pm 1,18)\end{array}$ & $\begin{array}{c}1,01 \text { a } \\
( \pm 0,17)\end{array}$ & $\begin{array}{c}0,39 \mathrm{~d} \\
( \pm 0,14)\end{array}$ \\
\hline Junho & $\begin{array}{l}4,68 \mathrm{~cd} \\
( \pm 0,46)\end{array}$ & $\begin{array}{l}0,40 \mathrm{bc} \\
( \pm 0,07)\end{array}$ & $\begin{array}{l}0,47 \mathrm{~d} \\
( \pm 0,08)\end{array}$ & $\begin{array}{l}8,28 \mathrm{ab} \\
( \pm 4,30)\end{array}$ & $\begin{array}{l}1,16 \text { ac } \\
( \pm 0,28)\end{array}$ & $\begin{array}{l}0,42 \mathrm{~cd} \\
( \pm 0,09)\end{array}$ \\
\hline Julho & $\begin{array}{l}4,75 \mathrm{~cd} \\
( \pm 0,42)\end{array}$ & $\begin{array}{c}0,44 \mathrm{abc} \\
( \pm 0,07)\end{array}$ & $\begin{array}{l}0,47 \mathrm{~d} \\
( \pm 0,21)\end{array}$ & $\begin{array}{l}8,45 \mathrm{ab} \\
( \pm 5,01)\end{array}$ & $\begin{array}{c}1,10 \mathrm{a} \\
( \pm 0,30)\end{array}$ & $\begin{array}{l}0,46 \mathrm{~cd} \\
( \pm 0,07)\end{array}$ \\
\hline Agosto & $\begin{array}{l}4,97 \mathrm{~cd} \\
( \pm 0,79)\end{array}$ & $\begin{array}{l}0,43 \mathrm{bc} \\
( \pm 0,09)\end{array}$ & $\begin{array}{c}0,71 \text { abcd } \\
( \pm 0,37)\end{array}$ & $\begin{array}{l}9,23 \text { a } \\
( \pm 5,37)\end{array}$ & $\begin{array}{c}1,24 \text { a } \\
( \pm 0,15)\end{array}$ & $\begin{array}{c}0,38 \mathrm{~d} \\
( \pm 0,08)\end{array}$ \\
\hline Setembro & $\begin{array}{c}5,43 \mathrm{abc} \\
( \pm 1,07)\end{array}$ & $\begin{array}{c}0,47 \mathrm{abc} \\
( \pm 0,09)\end{array}$ & $\begin{array}{c}0,69 \text { abcd } \\
( \pm 0,23)\end{array}$ & $\begin{array}{l}9,76 \mathrm{a} \\
( \pm 4,36)\end{array}$ & $\begin{array}{c}1,20 \mathrm{a} \\
( \pm 0,21)\end{array}$ & $\begin{array}{c}0,52 \mathrm{c} \\
( \pm 0,12)\end{array}$ \\
\hline Outubro & $\begin{array}{c}6,18 \text { a } \\
( \pm 0,80)\end{array}$ & $\begin{array}{c}0,59 \text { a } \\
( \pm 0,14)\end{array}$ & $\begin{array}{c}0,87 \text { a } \\
( \pm 0,40)\end{array}$ & $\begin{array}{l}9,47 \text { a } \\
( \pm 4,92)\end{array}$ & $\begin{array}{c}1,33 \text { a } \\
( \pm 0,24)\end{array}$ & $\begin{array}{c}0,65 \mathrm{~b} \\
( \pm 0,17)\end{array}$ \\
\hline Novembro & $\begin{array}{c}6,08 \text { a } \\
( \pm 1,01)\end{array}$ & $\begin{array}{c}0,48 \text { abc } \\
( \pm 0,11)\end{array}$ & $\begin{array}{l}0,81 \mathrm{ab} \\
( \pm 0,28)\end{array}$ & $\begin{array}{l}9,28 \text { a } \\
( \pm 4,60)\end{array}$ & $\begin{array}{c}1,40 \mathrm{a} \\
( \pm 0,30)\end{array}$ & $\begin{array}{l}0,41 \mathrm{~cd} \\
( \pm 0,15)\end{array}$ \\
\hline Dezembro & $\begin{array}{c}6,16 \text { a } \\
( \pm 0,99)\end{array}$ & $\begin{array}{c}0,36 \mathrm{c} \\
( \pm 0,15)\end{array}$ & $\begin{array}{c}0,58 \text { bcd } \\
( \pm 0,34)\end{array}$ & $\begin{array}{c}5,37 \mathrm{c} \\
( \pm 1,82)\end{array}$ & $\begin{array}{c}1,03 \text { a } \\
( \pm 0,41)\end{array}$ & $\begin{array}{l}0,47 \mathrm{~cd} \\
( \pm 0,18)\end{array}$ \\
\hline Janeiro & $\begin{array}{l}5,92 \mathrm{ab} \\
( \pm 1,14)\end{array}$ & $\begin{array}{l}0,52 \mathrm{ab} \\
( \pm 0,13)\end{array}$ & $\begin{array}{c}0,75 \text { abc } \\
( \pm 0,40)\end{array}$ & $\begin{array}{l}6,53 \mathrm{bc} \\
( \pm 1,12)\end{array}$ & $\begin{array}{c}1,24 \text { a } \\
( \pm 0,17)\end{array}$ & $\begin{array}{c}0,81 \text { a } \\
( \pm 0,40)\end{array}$ \\
\hline Fevereiro & $\begin{array}{c}5,51 \mathrm{abc} \\
( \pm 0,58)\end{array}$ & $\begin{array}{c}0,48 \text { abc } \\
( \pm 0,06)\end{array}$ & $\begin{array}{c}0,85 \text { a } \\
( \pm 0,13)\end{array}$ & $\begin{array}{c}7,53 \text { abc } \\
( \pm 1,76)\end{array}$ & $\begin{array}{c}1,33 \text { a } \\
( \pm 0,24)\end{array}$ & $\begin{array}{l}0,72 \mathrm{ab} \\
( \pm 0,36)\end{array}$ \\
\hline Março & $\begin{array}{c}5,06 \mathrm{bcd} \\
( \pm 1,62)\end{array}$ & $\begin{array}{c}0,44 \text { abc } \\
( \pm 0,14)\end{array}$ & $\begin{array}{c}0,70 \text { abcd }) \\
\quad( \pm 0,25)\end{array}$ & $\begin{array}{l}7,73 \mathrm{ab} \\
( \pm 2,48)\end{array}$ & $\begin{array}{c}1,29 \text { a } \\
( \pm 0,40)\end{array}$ & $\begin{array}{l}0,73 \mathrm{ab} \\
( \pm 0,35)\end{array}$ \\
\hline Média & 5,28 & 0,45 & 0,66 & 7,88 & 1,20 & $\mathbf{0 , 5 3}$ \\
\hline CV\% & 13,29 & 13,50 & 23,06 & 18,30 & 10,89 & 29,17 \\
\hline $\mathbf{r}_{1}{ }^{13}$ & 0,33 & 0,55 & $0,60 *$ & 0,54 & $0,68^{*}$ & 0,51 \\
\hline $\mathbf{r}_{2}$ & 0,37 & $-0,01$ & 0,39 & $-0,47$ & 0,41 & $0,66^{*}$ \\
\hline $\mathbf{r}_{3}$ & $\mathbf{- 0 , 7 2 * *}$ & $-0,55$ & $-0,62 *$ & $-0,35$ & $-0,32$ & 0,05 \\
\hline
\end{tabular}

${ }^{11}$ Médias seguidas pela mesma letra, na vertical, não diferem entre si, pelo teste de Tukey, a $5 \%$ de probabilidade de erro;

${ }^{12}$ Valores entre parênteses, referem-se ao desvio- padrão da média; ${ }^{13}$ Correlação de PEARSON entre teores de nutrientes e a pluviosidade $\left(r_{1}\right)$, temperatura média do $\operatorname{ar}\left(r_{2}\right)$ e deposição média mensal de serapilheira $\left(r_{3}\right)$. * Correlação significativa $(\mathrm{p}<0,05) ; \mathrm{e}^{* *}$ Correlação significativa $(\mathrm{p}<0,01)$.

Os teores de P mostraram-se variáveis, mas sem tendência de períodos com maior ou menor concentração, no período dos três anos de estudo. Os teores de Ca foram elevados no período de transição entre o inverno e a primavera (agosto a novembro). O S, no verão, esteve mais concentrado nas acículas, correlacionando-se com a temperatura média do ar $(\mathrm{r}=0,66$; e $\mathrm{p}<0,05)$. Os teores de $\mathrm{Mg}$ na serrapilheira foram estatisticamente iguais ( $p>0,05)$ durante todos os meses do ano, não apresentando comportamento definido, mas correlacionou-se com a pluviosidade $(r=0,68$; e $\mathrm{p}<0,05)$.

Em relação aos micronutrientes (Tabela 3), verificaram-se valores bastantes distintos durante os diferentes meses do ano, à exceção de B e Mn, que apresentaram coeficiente de variação abaixo de $20 \%$.
Nesse último elemento, os teores foram iguais estatisticamente $(p>0,05)$ em quase todos os meses do ano, exceto nos meses de setembro e dezembro.

A temperatura média do ar influenciou negativamente os teores de Fe, Mn e Zn na serapilheira, apresentando: $r=-0,73(p<0,01), r=-0,63$ e $r=-0,67(p<0,05)$, respectivamente. $\mathrm{O}$ teor de $\mathrm{Cu}$ correlacionou-se com a pluviosidade $(r=0,67$; e p < 0,05), e o $B$ não demonstrou correlação com as variáveis analisadas.

$\mathrm{O}$ aporte de macronutrientes (Figura 2) foi maior em Ca e N, os quais também apresentaram os maiores teores médios na serapilheira, correspondendo, juntamente, a cerca de $82 \%$ do total aportado ao solo, com magnitude de transferência na seguinte ordem decrescente: $\mathrm{Ca}>\mathrm{N}>\mathrm{Mg}>\mathrm{K}>\mathrm{S}>\mathrm{P}$.

R. Árvore, Viçosa-MG, v.34, n.1, p.85-94, 2010 

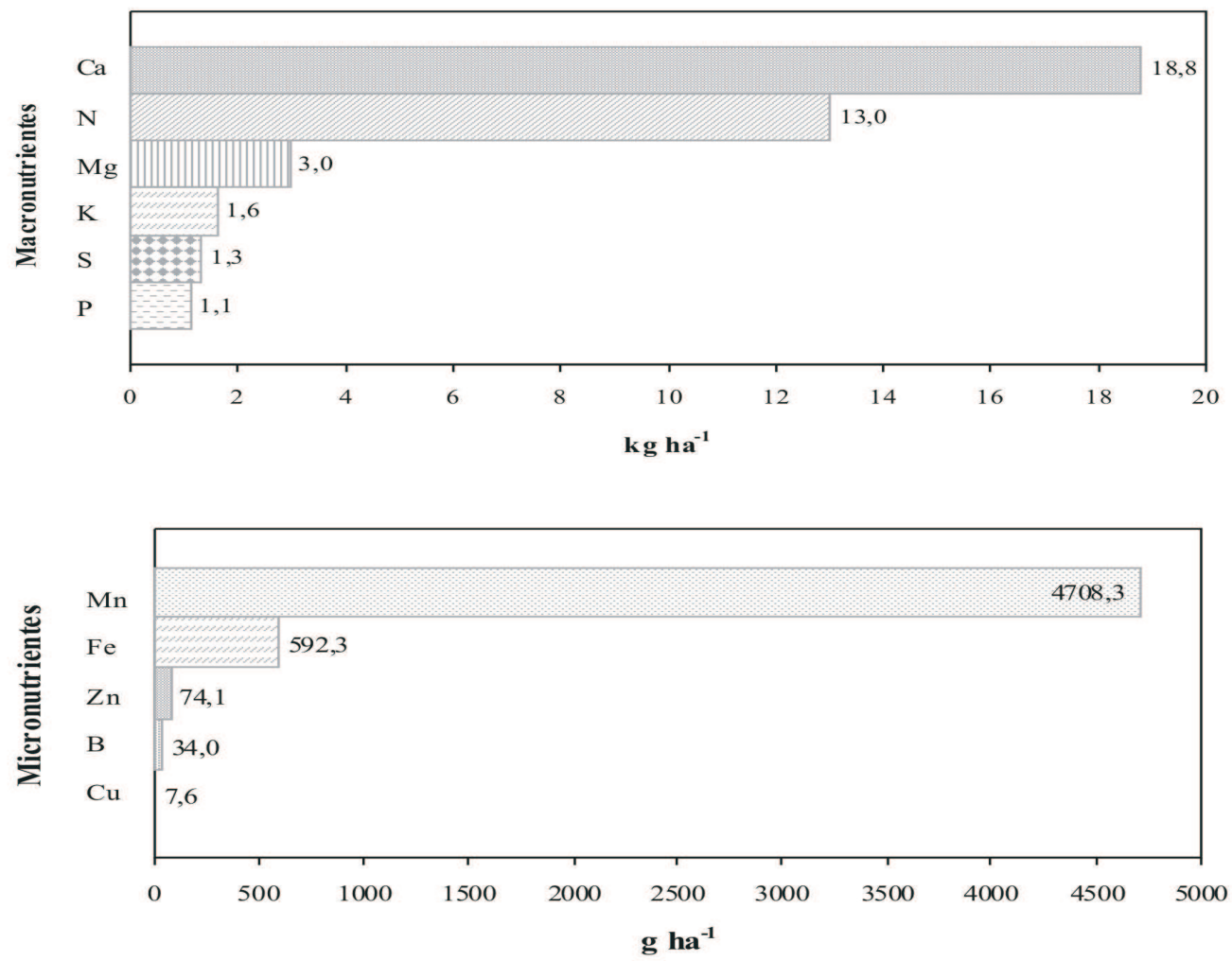

Figura 2- Quantidade média anual do aporte de nutrientes através da serapilheira em Pinus taeda L. implantado em campo nativo., Cambará do Sul, - RS.

Figure 2-Annual mean nutrient input through litterfall in Pinus taeda L. implanted in native grass., Cambará do Sul, - RS.

Tabela 3 - Teor médio mensal e anual de micronutrientes na serapilheira de um povoamento de Pinus taeda. Abr./2004 a Mar./2007. Cambará do Sul, RS.

Table 3 - Monthly and annually mean micronutrient content in Pinus taeda stand litter, from April 2004 to March, 2007. Cambará do Sul, RS.

\begin{tabular}{|c|c|c|c|c|c|}
\hline \multirow[t]{2}{*}{ Meses } & \multicolumn{5}{|c|}{ Micronutrientes $\left(\mathrm{mg} \mathrm{kg}^{-1}\right)$} \\
\hline & $\mathrm{B}$ & $\mathrm{Cu}$ & $\mathrm{Fe}$ & $\mathrm{Mn}$ & $\mathrm{Zn}$ \\
\hline Abril & $\begin{array}{c}13,39 \mathrm{abc}^{11} \\
( \pm 2,65)^{12}\end{array}$ & $\begin{array}{c}1,67 \mathrm{e} \\
( \pm 0,56)\end{array}$ & $\begin{array}{l}174,72 \mathrm{~d} \\
( \pm 59,48)\end{array}$ & $\begin{array}{c}1858,50 \mathrm{ab} \\
( \pm 604,82)\end{array}$ & $\begin{array}{c}30,67 \text { abcd } \\
( \pm 9,84)\end{array}$ \\
\hline Maio & $\begin{array}{c}14,61 \mathrm{ab} \\
( \pm 5,80)\end{array}$ & $\begin{array}{c}2,47 \mathrm{cde} \\
( \pm 0,35)\end{array}$ & $\begin{array}{c}219,55 \mathrm{~cd} \\
( \pm 41,41)\end{array}$ & $\begin{array}{c}1933,22 \mathrm{ab} \\
( \pm 482,48)\end{array}$ & $\begin{array}{c}26,20 \mathrm{bcd} \\
( \pm 8,97)\end{array}$ \\
\hline Junho & $\begin{array}{c}13,64 \mathrm{abc} \\
( \pm 4,15)\end{array}$ & $\begin{array}{l}3,14 \text { abcd } \\
( \pm 0,78)\end{array}$ & $\begin{array}{c}301,35 \mathrm{ab} \\
( \pm 74,04)\end{array}$ & $\begin{array}{c}2048,58 \mathrm{ab} \\
( \pm 864,14)\end{array}$ & $\begin{array}{c}34,83 \mathrm{abc} \\
( \pm 18,41)\end{array}$ \\
\hline Julho & $\begin{array}{c}12,49 \text { bc } \\
( \pm 2,83)\end{array}$ & $\begin{array}{c}4,05 \text { a } \\
( \pm 1,34)\end{array}$ & $\begin{array}{c}311,39 \mathrm{a} \\
( \pm 104,72)\end{array}$ & $\begin{array}{l}2114,03 \mathrm{ab} \\
( \pm 1159,57)\end{array}$ & $\begin{array}{c}34,29 \mathrm{abc} \\
( \pm 15,90)\end{array}$ \\
\hline Agosto & $\begin{array}{l}10,35 \mathrm{c} \\
( \pm 2,67)\end{array}$ & $\begin{array}{c}3,61 \text { abc } \\
( \pm 1,54)\end{array}$ & $\begin{array}{c}252,97 \mathrm{abcd} \\
( \pm 68,04)\end{array}$ & $\begin{array}{c}1735,32 \mathrm{ab} \\
( \pm 975,28)\end{array}$ & $\begin{array}{c}38,67 \mathrm{a} \\
( \pm 19,97)\end{array}$ \\
\hline Setembro & $\begin{array}{c}15,06 \mathrm{ab} \\
( \pm 6,38)\end{array}$ & $\begin{array}{l}3,80 \mathrm{ab} \\
( \pm 1,44)\end{array}$ & $\begin{array}{c}286,58 \mathrm{abc} \\
( \pm 57,80)\end{array}$ & $\begin{array}{l}2325,88 \text { a } \\
( \pm 998,41)\end{array}$ & $\begin{array}{l}36,90 \mathrm{ab} \\
( \pm 16,96)\end{array}$ \\
\hline Outubro & $\begin{array}{c}14,63 \mathrm{ab} \\
( \pm 3,37)\end{array}$ & $\begin{array}{c}4,27 \text { a } \\
( \pm 1,39)\end{array}$ & $\begin{array}{c}291,88 \text { abc } \\
( \pm 117,45)\end{array}$ & $\begin{array}{l}2267,78 \mathrm{ab} \\
( \pm 1104,38)\end{array}$ & $\begin{array}{l}37,32 \text { ab } \\
( \pm 21,39)\end{array}$ \\
\hline Novembro & $\begin{array}{r}16,22 \text { a } \\
( \pm 5,45) \\
\end{array}$ & $\begin{array}{c}3,01 \text { abcd } \\
-( \pm 1,34)\end{array}$ & $\begin{array}{c}226,13 \mathrm{bcd} \\
( \pm 67,38)\end{array}$ & $\begin{array}{l}2114,93 \mathrm{ab} \\
( \pm 1136,08)\end{array}$ & $\begin{array}{r}39,52 \text { a } \\
-( \pm 22,02) \\
\end{array}$ \\
\hline
\end{tabular}

R. Árvore, Viçosa-MG, v.34, n.1, p.85-94, 2010 
Tabela 3 - Cont.

\begin{tabular}{|c|c|c|c|c|c|}
\hline $\begin{array}{l}\text { Dezembro } \\
( \pm 5,48)\end{array}$ & $\begin{array}{c}14,32 \mathrm{ab} \\
( \pm 0,73)\end{array}$ & $\begin{array}{c}2,22 \text { de } \\
( \pm 69,65)\end{array}$ & $\begin{array}{c}176,33 \mathrm{~d} \\
( \pm 514,82)\end{array}$ & $\begin{array}{c}1264,07 \mathrm{~b} \\
( \pm 6,23)\end{array}$ & $19,63 \mathrm{~d}$ \\
\hline $\begin{array}{l}\text { Janeiro } \\
( \pm 3,94)\end{array}$ & $\begin{array}{c}12,76 \mathrm{abc} \\
( \pm 0,85)\end{array}$ & $\begin{array}{c}2,63 \text { bcde } \\
( \pm 75,20)\end{array}$ & $\begin{array}{l}207,21 \mathrm{~d} \\
( \pm 280,44)\end{array}$ & $\begin{array}{c}1572,95 \mathrm{ab} \\
( \pm 5,20)\end{array}$ & $25,71 \mathrm{bcd}$ \\
\hline $\begin{array}{l}\text { Fevereiro } \\
( \pm 4,44)\end{array}$ & $\begin{array}{c}12,15 \mathrm{bc} \\
( \pm 1,14)\end{array}$ & $\begin{array}{c}2,85 \text { bcde } \\
( \pm 30,65)\end{array}$ & $\begin{array}{c}247,70 \mathrm{abcd} \\
( \pm 357,31)\end{array}$ & $\begin{array}{c}1736,77 \mathrm{ab} \\
( \pm 6,75)\end{array}$ & $23,67 \mathrm{~cd}$ \\
\hline $\begin{array}{l}\text { Março } \\
( \pm 5,60)\end{array}$ & $\begin{array}{c}11,58 \mathrm{bc} \\
( \pm 1,73)\end{array}$ & $\begin{array}{l}3,64 \mathrm{abc} \\
( \pm 78,35)\end{array}$ & $\begin{array}{c}180,35 \mathrm{~d} \\
( \pm 587,37)\end{array}$ & $\begin{array}{c}1940,55 \mathrm{ab} \\
( \pm 8,04)\end{array}$ & $23,61 \mathrm{~cd}$ \\
\hline Média & 13,43 & 3,11 & 239,68 & 1909,38 & 30,92 \\
\hline CV\% & 12,29 & 25,29 & 20,86 & 15,79 & 22,24 \\
\hline $\mathbf{r}_{1}^{13}$ & 0,38 & $0,67 *$ & 0,26 & 0,41 & 0,19 \\
\hline $\mathbf{r}_{2}$ & 0,01 & $-0,46$ & $\mathbf{- 0 , 7 3} * *$ & $-0,63 *$ & $-0,67 *$ \\
\hline $\mathbf{r}_{3}$ & $-0,09$ & $-0,31$ & $-0,22$ & $-0,04$ & $-0,36$ \\
\hline
\end{tabular}

${ }^{11}$ Médias seguidas pela mesma letra, na vertical, não diferem entre si, pelo teste de Tukey, a 5\% de probabilidade de erro; ${ }^{12}$ Valores entre parênteses, referem-se ao desvio- padrão da média; ${ }^{13}$ Correlação de PEARSON entre teores de nutrientes e a pluviosidade $\left(r_{1}\right)$, temperatura média do $\operatorname{ar}\left(r_{2}\right)$ e deposição média mensal de serapilheira $\left(r_{3}\right)$ * Correlação significativa $(\mathrm{p}<0,05) ; \mathrm{e}^{* *}$ Correlação significativa $(\mathrm{p}<0,01)$.

Entre os micronutrientes (Figura 2), o Mn apresentou maior aporte, superando a maioria dos macronutrientes (P, S, K e Mg). Os elementos Mn e Fe foram responsáveis por cerca de $98 \%$ do total aportado ao solo através da queda da serapilheira, e, ainda, detectou-se que $\mathrm{o}$ aporte de micronutrientes foi decrescente na seguinte ordem: $\mathrm{Mn}>\mathrm{Fe}>\mathrm{Zn}>\mathrm{B}>\mathrm{Cu}$.

\section{DISCUSSÕES}

Em geral, ocorreram os maiores teores de nutrientes na serapilheira durante os meses de maior pluviosidade. Para Dias et al. (2002), a reposição hídrica (maior pluviosidade), poderia favorecer maior absorção de alguns nutrientes, colocando-os plenamente disponíveis para as plantas. Essa maior absorção, que é realizada pelas raízes das plantas, é devida, principalmente, ao fluxo de massa, o qual está diretamente relacionado com o volume de água, que forma a solução líquida do solo, na qual os nutrientes estão disponíveis (MALAVOLTA et al., 1997). Aumentando, dessa forma, a concentração desses elementos em todas as partes da planta, sobretudo nas folhas, onde ocorre a maior parte das atividades metabólicas (FERRI, 1985).

Intervenções realizadas nesses povoamentos, levando em consideração a redução da área foliar, através da prática silvicultural de desrama, poderiam, dessa forma, em períodos chuvosos ou de temperatura elevada, acarretar maior remoção de nutrientes das plantas, devido ao efeito de concentração no tecido foliar, como mencionado anteriormente.
À exceção do S, B e Mn da serapilheira, verificaram-se, com relação aos demais elementos, maiores teores nos períodos de menor deposição da serapilheira (inverno-primavera), indicando efeito de concentração devido à baixa produção de serapilheira e, possivelmente, por causa da maior disponibilidade hídrica no período. O mesmo foi verificado por Bertalot et al. (2004) em diferentes espécies de leucenas.

A temperatura média do ar influenciou, de forma negativa, o aumento dos teores de Fe, Mn e Zn e, positiva, o S, ambos significativamente. De forma geral, os macronutrientes foram afetados pela elevação da temperatura, causando aumento nos seus teores, à exceção de $\mathrm{P}$ e Ca. O P não apresentou nenhuma relação com a variação da temperatura, enquanto o Ca e os micronutrientes (exceto B) sofreram decréscimo nos seus teores com o aumento da temperatura.

Isso poderia ser atribuído ao fato de que o aumento da temperatura desencadeia uma série de eventos fisiológicos na planta, havendo maior demanda por nutrientes para suprir suas necessidades vitais, em virtude do crescimento acelerado que se estabelece no vegetal devido à elevação da temperatura (MALAVOLTA et al., 1997; EPSTEIN e BLOOM, 2006; FONTES, 2006).

Entre todos os nutrientes, o Ca foi o que apresentou o maior teor. Segundo Clevelário Jr. (1996 apud BORÉM e RAMOS, 2002), o enriquecimento em cálcio na serapilheira pode ser decorrente de liberação mais lenta desse elemento pelo material recém-caído, da

R. Árvore, Viçosa-MG, v.34, n.1, p.85-94, 2010 
retranslocação de outros elementos antes da abscisão e, ou, consequência da retenção de Ca contido na transprecipitação (chuva que atravessa o dossel) para a serapilheira.

O Ca é elemento que faz parte da lamela média da parede celular (MALAVOLTA et al., 1997), por isso é considerado imóvel na planta, o que faz que ele fique armazenado em forma de cristais na folha e permaneça assim, mesmo após a sua senescência (NEVES et al., 2001; DIAS et al., 2002).

Os teores de $\mathrm{P}, \mathrm{K}$ e $\mathrm{Mg}$ encontrados na serapilheira foram baixos, devido à baixa disponibilidade desses elementos no solo (Tabela 1), em consequência da elevada acidez do solo, o que afeta a disponibilização de nutrientes para a absorção pelas raízes da planta. Segundo Borém e Ramos (2002), a quantidade de Ca, Mg, K e P contida na serapilheira é comparável à quantidade disponível desse elemento no solo, principalmente nos primeiros $10 \mathrm{~cm}$. Contudo, cabe salientar que K e Mg podem sofrer lixiviação, o que contribui para explicar os baixos teores desses elementos na serapilheira.

Teores elevados de Fe e Mn são decorrentes das altas concentrações desses elementos no solo (Tabela 1), os quais podem causar toxidez às espécies vegetais. Contudo, povoamentos de pinus apresentam como característica desejável adaptação a solos pobres em nutrientes e com presença de $\mathrm{Al}^{3+} \mathrm{e}$, ainda, consegue apresentar bom crescimento, o que pode se favorecido se realizadas práticas de correção e de adubação do solo.

Os maiores aportes de nutrientes na serapilheira foram observados em $\mathrm{Ca}, \mathrm{N}$ e $\mathrm{Mn}$, refletindo maior disponibilidade desses no solo (Tabela 1). No estudo realizado por Melo e Resck (2002) com procedências de Pinus caribaea com 16 anos de idade e Koehler (1989), em três sítios diferentes (bom, médio e ruim) de Pinus taeda, esses autores encontraram tendência similar com os resultados deste trabalho, diferindo apenas o aporte de $\mathrm{N}$, que foi superior ao de $\mathrm{Ca}(\mathrm{N}>\mathrm{Ca}>\mathrm{K}>\mathrm{Mg}>$ $\mathrm{S}>\mathrm{P}$ ), sendo essa sequência constatada por Haag (1985).

Kolm (2001), estudando a ciclagem de nutrientes em plantações de Eucalyptus grandis, Koehler e Reissmann (1992) e Schumacher et al. (2004) em florestas de Araucaria angustifolia; e Oliveira Filho (1987) em Floresta Estacional, relataram a mesma magnitude deste trabalho, em transferência anual de macronutrientes ao solo via serapilheira.
Já com relação aos micronutrientes, Melo e Resck (2002), estudando procedências diferentes de Pinus caribaea com 16 anos de idade, encontraram a seguinte tendência: $\mathrm{Fe}>\mathrm{Mn}>\mathrm{Zn}>\mathrm{Cu}$; o mesmo foi relatado por Dias et al. (2002) em uma Floresta Estacional Semidecidual. Essa sequência difere da constatada neste estudo apenas quanto ao aporte de Mn, o qual foi superior ao de Fe.

\section{CONCLUSÕES}

Os teores de nutrientes na serapilheira diferiram entre os diferentes meses do ano $(p<0,05)$, com exceção do $\mathrm{Mg}$, e foram influenciados, significativamente, pela pluviosidade $(\mathrm{K}, \mathrm{Mg}$ e $\mathrm{Cu})$, temperatura do ar ( $\mathrm{S}, \mathrm{Fe}$, Mn e Zn) e quantidade de litter depositado ( $\mathrm{N} \mathrm{e} \mathrm{K}$ ).

Entre os macro e micronutrientes, Ca e Mn foram, respectivamente, aqueles que apresentaram maiores teores e quantidades entre os nutrientes que retornaram ao solo via deposição da serapilheira.

$\mathrm{O}$ aporte de nutrientes encontrados neste estudo demonstrou a importância da manutenção da serapilheira sobre o solo, do sítio florestal, para a continuidade da ciclagem de nutrientes.

\section{AGRADECIMENTOS}

Ao Conselho Nacional de Desenvolvimento Científico e Tecnológico (CNPq), pela bolsa de Iniciação Científica concedida ao primeiro autor; e á empresa Reflorestadores Unidos S/A, pela manutenção da área experimental, coleta, pelo envio de amostras e auxílio logístico.

\section{REFERÊNCIAS}

BALIEIRO, F. C. et al. Dinâmica da serapilheira e transferência de nitrogênio ao solo, em plantios de Pseudosamanea guachapele e Eucalyptus grandis. Pesquisa Agropecuária Brasileira, v.39, n.6, p.597-601,2004.

BERTALOT, M. J. A. et al. Retorno de nutrientes ao solo via deposição de serapilheira de quatro espécies leguminosas arbóreas na região de Botucatu-São Paulo, Brasil. Revista Scientia Florestalis, n.65, p.219227,2004.

BORÉM, R. A. T.; RAMOS, D. P. Variação estacional e topográfica de nutrientes na serapilheira de um fragmento de mata atlântica. Revista Cerne, v.8, n.2, p.42-59, 2002. 
BRASIL. Ministério da Agricultura. Divisão de Pesquisa Pedológica DNPEA. Levantamento de reconhecimento dos solos do Estado do Rio Grande do Sul. Recife: 1973. 431p. (Boletim Técnico, 30).

BRITEZ, R. M. et al. Deposição estacional de serapilheira e macronutrientes em uma Floresta de Araucária, São Mateus do Sul, Paraná. In: CONGRESSO NACIONAL SOBRE ESSÊNCIAS NATIVAS, 2., 1992, São Paulo; Revista do Instituto Florestal, v.4, n.3, p.766-772, 1992.

DIAS, H. C. T. et al. Variação temporal de nutrientes na serapilheira de um fragmento de floresta estacional semidecidual em Lavras, Minas Gerais - Brasil. Revista Cerne, v. 8 , n.2, p. 1-16, 2002.

\section{EPSTEIN, E.; BLOOM, A. J. Nutrição} mineral de plantas: princípios e perspectivas. Tradução Maria Edna Tenório Nunes. Londrina: Planta, 2006. 403p.

FERREIRA, C. A. et al. Pesquisa sobre nutrição de pinus no sul do Brasil. Revista da Madeira, v.14, n.83, 2004.

FERRI, M. G. Fisiologia vegetal 1. 2.ed. São Paulo: EPU, 1985. 362p.

FONTES, P. C. R. Diagnóstico do estado nutricional das plantas. Viçosa, MG: Universidade Federal de Viçosa, 2006. 122p.

GOLLEY, F. B. Nutrient cycling and nutrient conservation. In: Tropical forest ecosystems: structure and function. Amsterdam: Elsevier, 1983. p.137-156.

GONZÁLEZ, M. I. M.; GALLARDO, J. F. El efecto hojarasca: una revisión. Anales de Edafología y Agrobiología, v.41, p.1130-1157, 1982.

HAAG, H. P. Ciclagem de nutrientes em florestas tropicais. Campinas: Fundação Cargill, 1985. 144p.

JORDAN, C. F. Ciclagem de nutrientes e silvicultura de plantações na Bacia Amazônica. In: REUNIÃO BRASILEIRADE FERTILIDADE DO SOLO, 16., 1985, Ilhéus. Anais... Ilhéus: 1985. p.187-202.
KOEHLER, C. W.; REISSMANN, C. B.

Macronutrientes retornados com a serapilheira Araucaria angustifolia em função do sítio. In: CONGRESSO NACIONAL SOBRE ESSÊNCIAS NATIVAS, 2., 1992, São Paulo: Revista do Instituto Florestal, v.4, p.645-648, 1992. (Edição Especial).

KOEHLER, C. W.; REISSMANN, C. B.; KOEHLER, H. S. Deposição de resíduos orgânicos (serapilheira) e nutrientes em plantio de Araucaria angustifolia em função do sítio. Revista do Setor de Ciências Agrárias, v.9, n.1, p.89-96, 1987.

KOEHLER, W. C. Variação estacional de deposição de serapilheira e de nutrientes em povoamentos de Pinus taeda na região de Ponta Grossa-PR. 1989. 138f. Tese (Doutorado em Ciências Florestais) - Universidade Federal de Paraná, Curitiba, 1989.

KOLM, S. Ciclagem de nutrientes e variações do microclima em plantações de Eucalyptus grandis Hill ex Maiden manejadas através de desbastes progressivos. 2001. 73f. Dissertação (Mestrado em Recursos Florestais). Escola Superior de Agricultura Luiz de Queiroz, Piracicaba, 2001.

MALAVOLTA, E.; VITTI, G. C.; OLIVEIRA, S. A. Avaliação do estado nutricional das plantas: princípios e aplicações. 2. ed. Piracicaba: Associação Brasileira para a Pesquisa da Potassa e do Fosfato, 1997. 319p.

MELO, J. T.; RESCK, D. V. S. Retorno, ao solo, de nutrientes de serapilheira de pinus no cerrado do Distrito Federal. Planantina, DF: Embrapa Cerrados, 2002. 18p. (Boletim de Pesquisa e Desenvolvimento/Embrapa Cerrados, 75)

MOREnO, J. A. Clima do Rio Grande do Sul. Porto Alegre: Secretaria da Agricultura, 1961. 41p.

MOTTA, F. S.; BEISDORF, M. J. C.; GARCEZ, R. B. Zoneamento agrícola do Rio Grande do Sul e Santa Catarina: normas agro-climáticas. Pelotas: Ministério da Agricultura, 1971. 80p.

NEVES, E. J. M.; MARTINS, E. G.; REISSMANN, C. B. Deposição de serapilheira e de nutrientes de duas espécies da Amazônia. Boletim de Pesquisa Florestal, n.43, p.47-60, 2001. (Edição Especial).

R. Árvore, Viçosa-MG, v.34, n.1, p.85-94, 2010 
OLIVEIRA FILHO, R. R. Produção e decomposição de serapilheira no Parque Nacional da Tijuca, RJ. 1987. 107f. Dissertação (Mestrado em Geografia) Departamento de Geografia. Instituto de Geociências, Rio de Janeiro, 1987.

POGGIANI, F.; SCHUMACHER, M. V. Ciclagem de nutrientes em florestas nativas. In: GONÇALVES, J. L.; BENEDETTI, V. (Eds.) Nutrição e fertilização florestal. Piracicaba: IPEF, 2000. p.285-306.

PRITCHeTt, W. L. Properties and management of forest soils. New York: John Wiley, 1979. 500p.

SANCHEZ, P. A. Properties and management of soils in the tropics. New York: John Wiley and Sons, 1976. 409p.

SCHUMACHER, M. V. et al.

Monitoramento das características físicas e químicas do solo em área de campo nativo, florestada com Pinus taeda L. Santa Maria, Universidade Federal de Santa Maria, 2007. 29p. (Relatório Parcial de Pesquisa).

SCHUMACHER, M. V. et al. Produção de serapilheira em uma floresta de Araucaria angustifolia (bertol.) kuntze no município de Pinhal Grande-RS. Revista Árvore, v.28, n.1, p.29-37, 2004.
SCHUMACHER, M. V. et al. Retorno de nutrientes via deposição de serapilheira em um povoamento de acácia-negra (Acacia mearnsii De Wild.) no estado do Rio Grande do Sul. Revista Árvore, v.27, n.6, p.791-798, 2003.

SCHUMACHER, M. V. Aspectos da ciclagem de nutrientes e do microclima em talhões de Eucalyptus camaldulensis Dehnh, Eucalyptus grandis Hill ex Maiden e Eucalyptus torrelliana F. Mesell. 1992. 87f. Dissertação (Mestrado em Engenharia Florestal) - Escola Superior de Agricultura “Luiz de Queiroz”, Piracicaba, 1992.

SOARES, C. P. B.; NETO, F. P.; SOUZA, A. L. Dendrometria e inventário florestal. Viçosa, MG: Universidade Federal de Viçosa, 2006. 276p.

SPSS. Statistical package for the social sciences: programa de computador, ambiente windows. Versão 7.5.1. Chicago: 1996.

STRECK, E. V. et al. Solos do Rio Grande do Sul. Porto Alegre: Emater-RS/UFRGS, 2002. 107p.

TEDESCO, M. J. et al. Análise de solo, plantas e outros materiais. Porto Alegre: Universidade Federal do Rio Grande do Sul, 1995. 118p. (Boletim técnico, n.5).

VITAL, A. R. T. Produção de serapilheira e ciclagem de nutrientes de uma Floresta Estacional Semidecidual em Zona Ripária. Revista Árvore, v.28, n.6, p.793-800, 2004. 